\title{
INFORMATION ET DÉSINFORMATION DANS LA PRESSE AU DÉBUT DE L'ÈRE MEIJI
}

\section{Christiane Séguy}

RÉSUMÉ: Manipulation de l'information pendant la Restauration de Meiji (Meiji ishin) et la guerre du sud-ouest (seinan sensô). Législation de presse au début de l'ère Meiji. Attitude des journaux en période de crise et problèmes de l'équilibre entre morale, censure et démagogie.

RESUMO: A manipulação da informação durante a Restauração de Meiji (Meiji ishin) e a guerra do sudoeste (seinan sensô). A legislação da imprensa no início da época Meiji. A atitude dos jornais em período de crise e os problemas de equilíbrio entre a moral, a censura e a demagogia.

PALAVRAS-CHAVE: informação; jornais; Meiji ishin; seinan sensô; legislação da imprensa.

MOTS CLÉS: information; journaux; Meiji ishin; seinan sensô; législation de presse.

A diverses reprises au cours de son histoire, la presse japonaise s'est développée à la faveur d'événements exceptionnels: guerres, catastrophes ou troubles sociaux. Mais tandis que les techniques s'améliorent et se multiplient pour répondre au besoin croissant d'information en période de crise, se pose la question délicate de la manipulation de cette information. La guerre du Golfe, le tremblement de terre de Kobe, l'affaire de la secte Aum ou divers scandales politico-financiers nous rappellent que les problèmes de l'équilibre délicat entre morale, censure et surenchère démagogique sont très actuels. Mais qu'en est-il au début de l'époque Meiji, qui voit la naissance et le développement de la presse en même temps que la création d'une législation sévère, le plus souvent dictée par les événements? C'est ce que nous nous proposons d'examiner. 
Entre le printemps et l'été 1868 , brève période au cours de laquelle les troupes impériales quittent Kyôto et marchent sur la future capitale, on voit surgir des sortes de petits cahiers brochés diffusés principalement à Edo. Tous défendent le $B a k u f u$ et dénigrent sans exception les fiefs du sud-ouest. Bien plus, ils annoncent des victoires là où manifestement les troupes shôgunales avaient essuyé des défaites. Quelle est la part d'impact du Bakufu, et quelle est celle du manque d'informations fiables? L'un des éditeurs de ces brochures, Kishida Ginkô (1833-1905), note à ce sujet:

Les gens avides de nouvelles de la guerre se disputaient les journaux [...] ceux-ci transmettaient ce qui se disait de bouche à oreille, ou bien, comme ils savaient que cela ne plaisait pas au public d'Edo de lire toujours que les troupes de l'ouest étaient vainqueurs, ils écrivaient parfois exprès qu'elles avaient perdu [...] ainsi les ventes augmentaient prodigieusement et les revendeurs faisaient de gros bénéfices (in: Yamamoto, 1948, 2526).

Mais si Ginkô était un éditeur indépendant, la plupart des éditeurs de ces journaux avant la lettre étaient liés au Bakufu: Yanagawa Shunsan (1832-1870), par exemple, qui était professeur depuis 1864 au Kaiseijo, "Institut pour le développement des connaissances", ou Fukuchi Gen'ichirô [Ôchi] (1841-1906), traducteur et interprète officiel depuis 1859. Notons encore qu'à cette époque, le journal, au sens actuel du terme n'était pas encore né, et que seuls quelques chercheurs du Bakufu en contact avec les langues occidentales en connaissaient l'existence et tentèrent de l'exploiter. Il semblerait toutefois que le parti pris pour le camp shôgunal $n$ ait pas résulté de pressions politiques, mais soit le fruit des convictions personnelles des auteurs. Par ailleurs, les quelques bulletins du camp impérial publiés dans le Kansai, rédigés dans un style sec et administratif, n'eurent aucun écho auprès de la population.

\section{Mise en place d'une législation de presse et premières scissions}

Après l'entrée de l'armée impériale à Edo, toutes les publications proshôgunales seront interdites. Le nouveau gouvernement éditera le Dajôkan nisshi, "Bulletin du Conseil politique suprême" et quelques autres bulletins officiels très éphémères, et l'année suivante, le 20 mars 1869 , est promulguée la première réglementation de presse du Japon, Shinbunshi inkô jôrei, "Ordonnance relative à la publication des journaux". Celleci met fin à la période d'interdiction et autorise la publication de journaux selon des critères précis: autorisation préalable, responsabilité de l'éditeur pour le contenu, et restriction des sujets publiables. Le nouveau gouvernement espère ainsi susciter la création de journaux favorables à sa politique et aptes à propager ses nouvelles idées de modernisation du bunmei kaika. Cet objectif sera atteint, surtout après la promulgation de la nouvelle réglementation de presse, Shinbun jôrei, du 3 septembre 1871, qui constitue un véritable encouragement à la publication (exemple l'article 1: "les journaux doivent 
avoir pour objectif de développer les connaissances des hommes"). La même année et l'année suivante sont créés les premiers quotidiens de type occidental au Japon: Yokohama mainichi shinbun (28.1.1871), Tôkyô nichi nichi shinbun (1872, ancêtre du Mainichi shinbun), ou Yûbin hôchi shinbun (actuel Hôchi shinbun), suivis des premiers journaux de province.

Ces journaux, tout comme les premières revues qui voient le jour pendant cette période, paraissent souvent avec l'aide directe ou indirecte du gouvernement et restent fidèles à la nouvelle orientation jusqu'à l'éclosion d'un débat qui va diviser la classe dirigeante, à propos de la mise à la raison de la Corée, Seikanron, en octobre 1873. Durant l'absence des membres de la mission Iwakura en Europe, ceux qui conduisent les affaires - Saigô Takamori, Gotô Shôjirô, Itagaki Taisuke et Eto Shinpei - mettent en préparation une intervention militaire en Corée, dans l' idée d'appliquer à la Corée les conditions fixées au Japon par les alliés occidentaux. Mais Iwakura Tomomi, Ôkubo Toshimichi et Kido Takayoshi [Kô'in], dès leur retour, les contraignent à abandonner leur projet.

Si le conflit n'a pas eu lieu, les journaux font à cette occasion preuve d'une attitude entièrement nouvelle: ils commencent à s'intéresser de près aux affaires de l'état et entament de violentes polémiques, rompant ainsi avec leur image liée à la "connaissance et au progrès" au service du gouvernement pour se transformer en une presse d'opinion. Cet événement décisif, au cours duquel les journaux se disputent largement, aboutira à la démission des quatre partisans de l'intervention, le 22 octobre 1873. Trois mois plus tard, Itagaki, Eto et Gotô signent le Minsen gi'in setsuritsu kenpakusho, "Manifeste pour la mise en place d'une assemblée élue par le peuple" (17 janvier 1874), qui met le feu aux poudres de ce qui sera le vaste mouvement Jiyûminken undô, le "Mouvement pour la liberté et les droits du citoyen" Mais dès le 19 octobre, prévoyant que l'opinion s'enflammerait, le gouvernement prend des mesures sévères de contrôle de la presse. Le Shinbunshi jômoku de 1873 remplace 1'ordonnance si encourageante de 1871 et reprend, de manière renforcée, celle de 1869: restriction des thèmes autorisés, interdiction de juger la politique intérieure et étrangère, obligation de discrétion des fonctionnaires. Cette réglementation n'empêche toutefois pas les journaux de se lancer dans la campagne pour le mouvement libéral. Tandis que tous les journaux de l'époque vont rallier le minkenha, "camp des droits du citoyen", seul le Tôkyô nichi nichi shinbun, dirigé par Fukuchi Gen'ichirô (qui a effectué un remarquable revirement d'opinion depuis Meiji), rallie le kanken-ha, le "camp du gouvernement", et sera dorénavant un fidèle défenseur de la politique officielle.

L'expédition punitive à Taïwan en juillet 1874 est un bref intermède qui n'est pas véritablement contesté par la presse, et serait presque passé inaperçu tant les préoccupations de poilitique intérieure sont prédominantes. Ôkubo Toshimichi, qui était pourtant contre la mise à la raison de la Corée, décide de lancer des troupes à Formose malgré l'opposition de Kido. Cette première campagne étrangère est l'occasion du premier déplacement d'un correspondant de guerre à l'étranger, celui de Kishida Ginkô, journaliste au Tôkyô nichi nichi shinbun de 1873 à 1877. 
Les longs mois de la guerre du sud-ouest ou soulèvement de Satsuma (février à septembre 1877), jalonnés de péripéties - répression de différents soulèvements antigouvernementaux dans les provinces du sud-ouest, refoulement de l'armée de Saigô Takamori obligée de se replier à Kagoshima, assaut final de l'armée gouvernementale et suicide de Saigô -, permettent d'analyser plus en détail l'attitude des journaux et des journalistes face à ce conflit de portée nationale. De nouveaux titres ont vu le jour depuis l'apparition des premiers quotidiens en 1871-1872; ils se répartissent en deux catégories: les $\hat{o}$-shinbun, grands journaux politiques à audience relativement limitée, et les petits journaux populaires, ko-shinbun, qui ne s'intéressent pas à la politique mais connaissent en revanche un grand succès. Parmi ces derniers, seul le Yomiuri shinbun, créé en 1874, se distingue par la variété de ses sujets (y compris politiques) et une recherche de qualité tant par la forme que par le fond.

La première difficulté à laquelle la jeune presse japonaise n'est pas encore préparée est celle de se procurer des nouvelles fiables de si loin et de les transmettre le plus rapidement possible à Tôkyô. Les quotidiens font habituellement appel à des informateurs, souvent des lecteurs (le Yûbin hôchi shinbun avait inauguré ce système), ou envoient des tanbôsha, collecteurs d'information sans qualification, chargés de transmettre les nouvelles aux rédacteurs à Tôkyô. Mais là, pour la première fois, les grands journaux sont placés en situation de concurrence. Ils envoient donc sur place leurs meilleurs éléments, voire leurs directeurs en personne: Fukuchi Ochi pour le Tokyô nichi nichi shinbun, Yano Fumio et Inukai Tsuyoshi pour le Yûbin hôchi shinbun, Narushima Ryûhoku (à Kyôto) pour le Chôya shinbun, et Takahashi Ki'ichi et Aeba Kôson pour le Yomiuri shinbun.

Fukuchi Ochi est alors quasiment considéré comme un rapporteur officiel; il réfêre même de la situation devant l'Empereur, et le prestige de son journal n'en fait qu'augmenter. Mais curieusement, dans cette affaire, tous les journaux du minken-ha (Chôya shinbun, Tôkyô akebono shinbun, Yûbin hôchi shinbun) se rangent également aux côtés du gouvernement. D'abord, sans le vouloir, parce que malgré leurs efforts, les quotidiens de la capitale, et plus encore de province, dépendent tout de même largement des dépêches gouvernementales. Ensuite parce que les journaux étaient soumis depuis 1875 à une législation sévère: l'ordonnance de presse shinbunshi jôrei, et la loi sur la diffamation et la calomnie zanbôritsu (décrets du Dajôkan du 28.6.1875). La période de 1875 à 1880 est d'ailleurs jalonnée d'un tel nombre d'amendes, d'interdictions ou suspensions de publication et d'emprisonnements de journalistes, qu'elle a été surnommée shinbun kyôfujidai, "l'époque de la terreur pour les journaux" En 1877 vient s'ajouter un décret interdisant la publication de nouvelles non-fondées, et les articles concernant la guerre du sud-ouest sont dorénavant soumis à la censure avant publication.

Cependant, en dehors du contrôle exercé par le pouvoir et des difficultés d'accès direct aux nouvelles, d'autres motivations encore semblent expliquer l'attitude des journaux libéraux: en effet, Saigô Takamori avait perdu sa considération auprès de la plupart des intellectuels lors de son engagement pour la mise à la raison de la Corée. 
Bien qu'ayant démissionné en même temps qu'Itagaki et ayant également rejoint l'opposition, il ne défendait pas comme lui les idées libérales. Au contraire, il restait attaché aux valeurs et aux statuts d'une époque désormais révolue. Rebelle, il a donc été considéré à la fois comme provocateur par le gouvernement et comme ultra-conservateur par les libéraux. Ceci explique pourquoi certains journaux ne se contentent pas seulement de s'aligner sur la position du gouvernement, mais se laissent aller à la surenchère démagogique et désignent Saïgô comme un véritable "ennemi numéro un" Fukuzawa Yukichi écrit dans un essai intitulé Meiji jûnen teichû kôron (24.10.1877, Fukuzawa Yukichi shû, Meiji bungaku zenshû, vol. 8, 1966, p. 248) "Ils disent du mal de lui de façon ignoble, proferent des menaces inutiles, souvent des insultes ou des calomnies qui dépassent toute réalité [...] c'est à se demander si ces journalistes ne nourissent pas une vieille rancune personnelle envers Saigô".

La première conséquence de la guerre du sud-ouest est un regain d'intérêt de la part des lecteurs pour les journaux. Presque tous connaissent un essor sans précédent. De plus, des journaux à priori qualifiés de populaires et apolitiques, notamment le Yomiuri shinbun, se distinguent particulièrement par la rapidité et la fiabilité des informations, bien plus que ses confrères de la grande presse politique. Il réussit par exemple l'exploit de publier un numéro spécial sur la mort de Saigô le jour même de l'événement, alors qu'á l'époque, un télégramme officiel de Kyûshû mettait facilement une journée. Avant la guerre du sud-ouest, il détenait déjà le plus fort tirage parmi les petits journaux, mais après il prend la tête de tous les quotidiens.

Peut-être peut-on déjà déceler dans ces premières années de Meiji quelques traits carctéristiques de la presse actuelle: tendance à la surenchère, voire au sensationnalisme, consensus avec le gouvernement (volontaire ou forcé), mais aussi priorité à l'information, prépondérance des journaux variés et distrayants au détriment des journaux politiquement engagés.

\section{Bibliographie}

BAKUMATSU Meiji shinbun zenshû, "Collection complète des journaux de la fin du régime shôgunal à Meiji”, 5 vols., Taiseidô, Tôkyô, 1934-1935.

KAWARABAN / shinbun - Edo / Meiji sanbyaku jiken, "kawaraban et journaux - trois cents événements marquants d'Edo à Meiji”), 4 vols., Taiyô korekushon, Heibonsha, 1978.

MEIJI bunka shiryô sôsho, "Série de documents relatifs à la culture de Meiji" 13 vols., vol. XII: shinbun-hen, "Journaux" publié sous la direction de Nishida Taketoshi, Kazama shobô, 1972.

MEIJI bunka zenshû, "Collection complète sur la culture de Meiji" vol. 4: "Shinbunhen", "Journaux", Nihon hyôron shinsha, 1928, rééd. 1955.

MIYATAKE, Gaikotsu et NISHIDA, Taketoshi. Meiji shinbun zasshi kankeisha ryakuden, "Aperçus biographiques relatifs aux journaux et revues de Meiji”, Meiji Taishô Genron shiryô, vol. XX, Misuzu shobô, 1985.

NIHON shinbun-shi chihôbetsu, "Histoire de la presse japonaise par région", Nihon shinbun kyôkai, 1956.

NiSHIDA, Taketoshi.Meiji jidai no shinbun to zasshi, "Journaux et revues de l'ère Meiji”, Shibundô, Tôkyô, 1961. 
ONO, Hideo. Shinbun no rekishi, "Histoire de la presse", Tôkyôdô shuppan, Tôkyô, 1961.

SÉGUY, Christiane. Histoire de la presse japonaise, P.O.F., 1993.

UCHIKAWA, Yoshimi. Meiji nyûsu jiten. "Dictionnaire des nouvelles à l'époque Meiji", 4 vols., Mainichi komyunikêshonzu, 1984.

Yамамото, Fumio. Nihon shinbun shi, "Histoire de la presse japonaise”, Kokusai shuppan, Tôkyô, 1948.

YAMAMOTO, Taketoshi. Shinbun to Minshû - Nihongata shinbun no keiseikatei, "Le journal et le peuple: la mise en place d'une presse spécifiquement japonaise", Kinokuniya shoten, Tôkyô, 1973.

YOSHIHARA, Isao. Shakaihendô to communication - Nihon kindaika katei ni okeru communication no tenkai, "Transformation de la société et communication - le développement de la communication dans le processus de modernisation du Japon" in Communication shakaigaku, "Sociologie de la communication", publié sous la direction de SATo, Takeshi, Saiensu-sha, Tôkyô, 1985, pp. 49-102.

Christiane Séguy

Université des Sciences Humaines de Strasbourg (France) 